Supporting Information for:

\title{
Stabilized Arsenic(I) Iodide: A Ready Source of As-I and a Useful Reagent for the Generation of Clusters
}

Bobby D. Ellis and Charles L. B. Macdonald*

Department of Chemistry and Biochemistry, University of Windsor, Windsor, Ontario, Canada N9B 3P4

\section{Summary of Computational Details}

Calculations were performed using the Gaussian $98^{1}$ suite of programs on a dual Xeon processor Dell Precision workstation. All calculations were performed using either the B3LYP/6-31G(d) (6-31+G(d) for As) or B3PW91/6-311+G(d) levels of theory. ${ }^{2-4}$ Each model was fully optimized in the point group indicated; bond lengths are reported in $\AA$ and angles are given in degrees, where $\mathrm{E}$ is either $\mathrm{P}, \mathrm{N}$ or $\mathrm{C}$ and dmpe is 1,2bis(dimethylphosphino)ethane. Atomic charges (in atomic units) were calculated using the $\mathrm{NBO}^{5,6}$ method as implemented in Gaussian98 and orbital visualizations were done using MOLDEN. ${ }^{7}$ The occupancy of the $4 p_{x}$ orbital on the central As atom is given in units of electrons. The "back-bonding" from the central atom to the phosphine ligands is demonstrated by the decreased occupancy of the $4 p_{x}$ orbital and is manifested in the shape and relative energetic stabilization of the highest occupied molecular orbital (HOMO) in the model bearing phosphine substituents.

Tabular Summary of B3LYP/Gen Results for $\left[\mathrm{L}_{2} A s^{\mathrm{I}}\right]^{ \pm 1}$ Model Compounds:

\begin{tabular}{|c|c|c|c|c|c|c|c|c|c|}
\hline Model & Symm. & Energy (au) & $\mathrm{N}_{\text {imag }}$ & $\mathrm{E}_{\text {homo }}(\mathrm{au})$ & $\mathrm{E}_{\text {lumo }}(\mathrm{au})$ & As-E & E-As-E & $\begin{array}{c}\text { Occ. As } \\
4 \mathrm{p}_{\mathrm{x}}\end{array}$ & $\mathrm{Q}(\mathrm{As})$ \\
\hline$\left(\mathrm{H}_{3} \mathrm{P}\right)_{2} \mathrm{As}^{+}$ & $\mathrm{C}_{2 \mathrm{v}}$ & -2919.4320928 & 0 & -0.37861 & -0.22510 & 2.302 & 95.9 & 1.73806 & -0.12699 \\
\hline & & & & & & & & & \\
\hline$\left(\mathrm{H}_{3} \mathrm{~N}\right)_{2} \mathrm{As}^{+}$ & $\mathrm{C}_{2 \mathrm{v}}$ & -2346.2586011 & 0 & -0.34654 & -0.22660 & 2.088 & 93.0 & 1.96947 & 0.36710 \\
\hline & & & & & & & & & \\
\hline$\left(\mathrm{H}_{3} \mathrm{C}\right)_{2} \mathrm{As}^{-}$ & $\mathrm{C}_{2 \mathrm{v}}$ & -2313.2306187 & 0 & 0.05130 & 0.14275 & 2.030 & 94.6 & 1.92845 & -0.26606 \\
\hline
\end{tabular}

\section{Output Summary of B3LYP/Gen Results for $\left[\mathrm{L}_{2} A s^{\mathrm{I}}\right]^{ \pm 1}$ Model Compounds:}

$\left(\mathbf{H}_{3} \mathbf{P}\right)_{2} \mathbf{A s}^{+}$

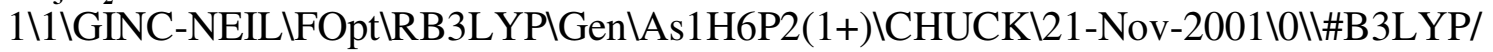

GEN OPT TEST \ldft optimization of (H3P)As(PH3) cation \1,1\As,0.,0,0.

8319314599\P, $1.7103944759,0 .,-0.7092600288 \backslash \mathrm{P},-1.7103944759,0 .,-0.70926$

$00288 \backslash \mathrm{H}, 2.8848249888,0 ., 0.0603022729 \backslash \mathrm{H}, 1.8931694526,-1.0992464322,-1.5$

$74135464 \backslash \mathrm{H}, 1.8931694526,1.0992464322,-1.574135464 \backslash \mathrm{H},-2.8848249888,0 ., 0$

$.0603022729 \backslash \mathrm{H},-1.8931694526,1.0992464322,-1.574135464 \backslash \mathrm{H},-1.8931694526$,

$-1.0992464322,-1.574135464 \mid I V e r s i o n=x 86-L i n u x-G 98 R e v A .11 .1 \backslash S t a t e=1-A 1 \backslash$

$\mathrm{HF}=-2919.4320928 \backslash \mathrm{RMSD}=4.277 \mathrm{e}-09 \backslash \mathrm{RMSF}=3.480 \mathrm{e}-05 \backslash \mathrm{Dipole}=0 ., 0 .,-1.6988458$

$\backslash \mathrm{PG}=\mathrm{C} 02 \mathrm{~V}[\mathrm{C} 2(\mathrm{As} 1), \mathrm{SGV}(\mathrm{H} 2 \mathrm{P} 2), \mathrm{X}(\mathrm{H} 4)] 1 \backslash @$

$\left(\mathrm{H}_{3} \mathbf{N}\right)_{2} \mathrm{As}^{+}$ 


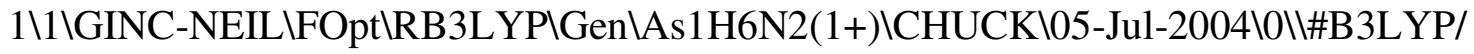
GEN OPT POP=(FULL,NBO) TEST\\dft optimization of $(\mathrm{H} 3 \mathrm{~N}) \mathrm{As}(\mathrm{NH} 3)$ cation $\backslash$ $1,1 \backslash \mathrm{As}, 0 ., 0 ., 0.5661583358 \backslash \mathrm{N}, 1.5146606962,0 .,-0.8708246429 \backslash \mathrm{N},-1.5146606$ $962,0 .,-0.8708246429 \backslash \mathrm{H}, 2.3812148611,0 .,-0.320621952 \backslash \mathrm{H}, 1.5491014153,-0$. 83447689,-1.4626090444\H,1.5491014153,0.83447689,-1.4626090444\Н,-2.38 $12148611,0 .,-0.320621952 \backslash \mathrm{H},-1.5491014153,0.83447689,-1.4626090444 \backslash \mathrm{H},-1$ $.5491014153,-0.83447689,-1.46260904441 \mid V e r s i o n=x 86-$ Linux-G98RevA.11.1 State=1-A1 $\backslash H F=-2346.2586011 \backslash R M S D=2.422 \mathrm{e}-09 \backslash \mathrm{RMSF}=9.529 \mathrm{e}-05 \backslash \mathrm{Dipole}=0 ., 0$. ,-2.3608691\PG=C02V [C2(As1),SGV(H2N2),X(H4)]॥\@

\section{$\left(\mathrm{H}_{3} \mathbf{C}\right)_{2} \mathrm{As}^{+}$}

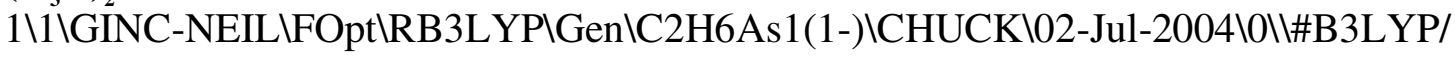
GEN OPT POP=(FULL,NBO) TESTIIdft optimization of (H3C)As(CH3) anionll$1,1 \backslash \mathrm{As}, 0 ., 0 ., 0.5151726819 \backslash \mathrm{C}, 1.491978936,0 .,-0.8619886809 \backslash \mathrm{C},-1.49197893$ $6,0 .,-0.8619886809 \backslash \mathrm{H}, 2.4502681923,0 .,-0.3253331852 \backslash \mathrm{H}, 1.4647108613,-0.8$ $916779298,-1.50154199 \backslash \mathrm{H}, 1.4647108613,0.8916779298,-1.50154199 \backslash \mathrm{H},-2.450$ $2681923,0 .,-0.3253331852 \backslash \mathrm{H},-1.4647108613,0.8916779298,-1.50154199 \backslash \mathrm{H},-1$ $.4647108613,-0.8916779298,-1.50154199 \|$ Version=x86-Linux-G98RevA.11.1 State $=1-\mathrm{A} 1 \backslash \mathrm{HF}=-2313.2306187 \backslash \mathrm{RMSD}=5.440 \mathrm{e}-09 \backslash \mathrm{RMSF}=4.451 \mathrm{e}-05 \backslash \mathrm{Dipole}=0 ., 0$. ,-1.0144615 $\backslash \mathrm{PG}=\mathrm{C} 02 \mathrm{~V}[\mathrm{C} 2(\mathrm{As} 1), \mathrm{SGV}(\mathrm{C} 2 \mathrm{H} 2), \mathrm{X}(\mathrm{H} 4)] \backslash \backslash @$

\section{Summary of B3PW91 Calculations on [( $\left.\left.\mathrm{Me}_{2} \mathrm{PCH}_{2} \mathrm{CH}_{2} \mathrm{PMe}_{2}\right) \mathrm{As}\right]^{+}$:}

\section{Optimized structures (in Z-matrix format) and energies for $\left[\left(\mathrm{Me}_{2} \mathrm{PCH}_{2} \mathrm{CH}_{2} \mathrm{PMe}_{2}\right) \mathrm{As}\right]^{+}$}

\section{Geometry Optimization of [(dmpe)As]}

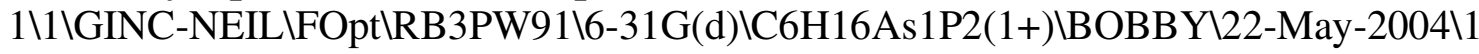
II\# B3PW91/6-31G(D) FOPT=Z-MATRIX FREQ=NORAMAN POP=(FULL,NBO) TEST $\backslash \backslash G e o m$ and Freq of DMPE-As+ $+\backslash 1,1 \backslash A s \backslash X, 1,1 . \mid P, 1$,pas,2,pasx $\backslash P, 1$, pas, 2,pasx,3 ,180.,0\C,3,cp1,1,cpas1,2,cpasx1,0\C,4,cp1,1,cpas1,2,cpasx1,0\C,3,cp2, 1,cpas2,2,cpasx2,0\C,4,cp2,1,cpas2,2,cpasx2,0\C,3,cp3,1,cpas3,2,cpasx3 ,0।C,4,cp3,1,cpas3,2,cpasx 3,0ไH,5,hc1,3,hcp1,1,hcpas1,0ไH,6,hc1,4,hcp1 ,1,hcpas1,0ไH,5,hc2,3,hcp2,1,hcpas2,0\H,6,hc2,4,hcp2,1,hcpas2,0\H,7,hc 3,3,hcp3,1,hcpas3,0\H,8,hc3,4,hcp3,1,hcpas3,0\H,9,hc4,3,hcp4,1,hcpas4,

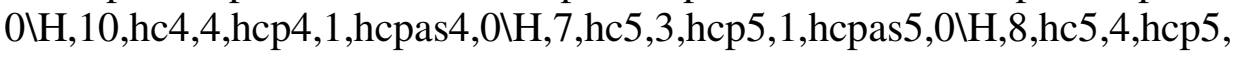
1,hcpas5,0\H,9,hc6,3,hcp6,1,hcpas6,0\H,10,hc6,4,hcp6,1,hcpas6,0\H,7,hc 7,3,hcp 7,1,hcpas7,0\H,8,hc7,4,hcp7,1,hcpas7,0\H,9,hc8,3,hcp8,1,hcpas8, $0 \backslash \mathrm{H}, 10, \mathrm{hc} 8,4, \mathrm{hcp} 8,1$,hcpas8,0 $\backslash$ pas $=2.26036451 \backslash$ pasx $=136.18117946 \backslash \mathrm{cp} 1=1.8$ 4664203 $\backslash$ cpas $1=107.21524521 \mathrm{lcpasx} 1=169.38540441 \mathrm{lcp} 2=1.82380186 \mathrm{lcpas} 2=11$ $3.46903393 \backslash \mathrm{cpasx} 2=50.69709035 \backslash \mathrm{cp} 3=1.82709955 \backslash \mathrm{cpas} 3=113.94938781 \backslash \mathrm{cpasx} 3$ $=-70.61954588 \mathrm{hc} 1=1.09635183 \mathrm{Vhcp} 1=109.67982979 \mathrm{Vhcpas} 1=157.90775637 \mathrm{Vhc} 2$ $=1.09576232 \mathrm{Vhcp} 2=106.83823753 \mathrm{lhcpas} 2=-85.92001978 \mathrm{lhc} 3=1.09315686 \mathrm{Vhcp} 3=$ $109.43675728 \mathrm{Vhcpas} 3=56.11402943 \mathrm{Vhc} 4=1.09535035 \mathrm{hcp} 4=111.35526451 \mathrm{Vhcpas}$ $4=177.21809786 \mathrm{hc} 5=1.09514741 \mathrm{hcp} 5=110.68824932 \mathrm{hcpas} 5=177.62685865 \mathrm{hc}$ $6=1.09336791 \mathrm{hcp} 6=109.61755975 \mathrm{hcpas} 6=-60.86841307 \mathrm{Vhc} 7=1.09446145 \mathrm{Vhcp} 7$ $=109.42863244 \mathrm{Vhcpas} 7=-62.49013179 \mathrm{hc} 8=1.09463681 \mathrm{Vhcp} 8=108.86035227 \mathrm{Vhcp}$ as8 $=56.90779551 \|$ Version $=x 86-$ Linux-G98RevA.11.1 $\backslash$ State $=1-A \backslash H F=-3154.379$ 
4209\RMSD $=3.458 \mathrm{e}-09 \backslash \mathrm{RMSF}=8.619 \mathrm{e}-06 \backslash \mathrm{Dipole}=0 ., 0 .,-1.7632217 \backslash \mathrm{PG}=\mathrm{C} 02[\mathrm{C} 2($ As1),X(C6H16P2)]川@

\section{Single Point Energy Calculation of [(dmpe)As $]^{+}$}

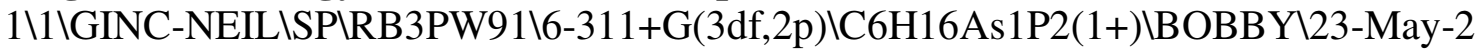
00410\॥ B3PW91/6-311+G(3DF,2P) GEOM=CHECK GUESS=READ SCF=TIGHT \#P

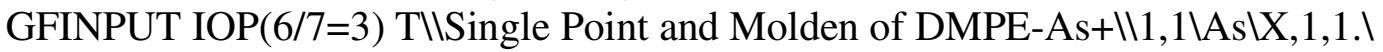
$\mathrm{P}, 1,2.2603645081,2,136.18117946 \backslash \mathrm{P}, 1,2.2603645081,2,136.18117946,3,180$. ,0\C,3,1.8466420282,1,107.21524521,2,169.385404411,0\C,4,1.8466420282,1 ,107.21524521,2,169.38540441,0\C,3,1.82380186,1,113.46903393,2,50.6970 9035,0।C $, 4,1.82380186,1,113.46903393,2,50.69709035,0 \backslash \mathrm{C}, 3,1.827099547,1$ ,113.94938781,2,-70.61954588,0\C,4,1.827099547,1,113.94938781,2,-70.61

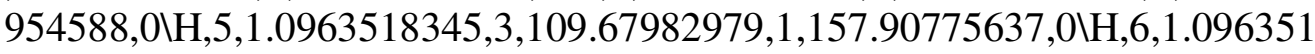
$8345,4,109.67982979,1,157.90775637,0 \backslash \mathrm{H}, 5,1.0957623197,3,106.83823753,1$ ,-85.92001978,0\H,6,1.0957623197,4,106.83823753,1,-85.92001978,0\H,7,1 $.0931568552,3,109.43675728,1,56.11402943,0 \backslash \mathrm{H}, 8,1.0931568552,4,109.4367$ $5728,1,56.11402943,0 \backslash \mathrm{H}, 9,1.0953503549,3,111.35526451,1,177.21809786,0 \backslash$ H,10,1.0953503549,4,111.35526451,1,177.21809786,0\H,7,1.0951474111,3,1 10.68824932,1,177.62685865,0\H,8,1.0951474111,4,110.68824932,1,177.626 $85865,0 \backslash \mathrm{H}, 9,1.0933679053,3,109.61755975,1,-60.86841307,0 \backslash \mathrm{H}, 10,1.093367$ $9053,4,109.61755975,1,-60.86841307,0 \backslash \mathrm{H}, 7,1.0944614548,3,109.42863244,1$ ,-62.49013179,0\H,8,1.0944614548,4,109.42863244,1,-62.49013179,0\H,9,1 $.0946368066,3,108.86035227,1,56.90779551,0 \backslash \mathrm{H}, 10,1.0946368066,4,108.860$ $35227,1,56.90779551,0 \| 1$ Version=x86-Linux-G98RevA.11.1।State $=1-\mathrm{AlHF}=-31$ 56.6510109\RMSD=6.293e-09\Dipole=0.,0.,-1.6997649\PG=C02 [C2(As1),X(C6 H16P2)]川@

(1) Gaussian 98, Frisch, M. J.; Trucks, G. W.; Schlegel, H. B.; Scuseria, G. E.; Robb, M. A.; Cheeseman, J. R.; Zakrzewski, V. G.; Montgomery, J. A., Jr.; Stratmann, R. E.; Burant, J. C.; Dapprich, S.; Millam, J. M.; Daniels, A. D.; Kudin, K. N.; Strain, M. C.; Farkas, O.; Tomasi, J.; Barone, V.; Cossi, M.; Cammi, R.; Mennucci, B.; Pomelli, C.; Adamo, C.; Clifford, S.; Ochterski, J.; Petersson, G. A.; Ayala, P. Y.; Cui, Q.; Morokuma, K.; Salvador, P.; Dannenberg, J. J.; Malick, D. K.; Rabuck, A. D.; Raghavachari, K.; Foresman, J. B.; Cioslowski, J.; Ortiz, J. V.; Baboul, A. G.; Stefanov, B. B.; Liu, G.; Liashenko, A.; Piskorz, P.; Komaromi, I.; Gomperts, R.; Martin, R. L.; Fox, D. J.; Keith, T.; Al-Laham, M. A.; Peng, C. Y.; Nanayakkara, A.; Challacombe, M.; Gill, P. M. W.; Johnson, B.; Chen, W.; Wong, M. W.; Andres, J. L.; Gonzalez, C.; Head-Gordon, M.; Replogle, E. S.; Pople, J. A., Gaussian Inc., Pittsburg, 2001.

(2) Becke, A. D. J. Chem. Phys. 1993, 98, 5648-5652.

(3) Lee, C. T.; Yang, W. T.; Parr, R. G. Phys. Rev. B 1988, 37, 785-789.

(4) Perdew, J. P.; Wang, Y. Phys. Rev. B 1992, 45, 13244-13249.

(5) NBO, Glendening, E. D.; Reed, A. E.; Carpenter, J. E.; Weinhold, R.

(6) Reed, A. E.; Curtiss, L. A.; Weinhold, F. Chemical Reviews 1988, 88, 899-926.

(7) MOLDEN, Shaftenaar, G., CAOS/CAMM Center Nijmegen:

Toernooiveld, Nijmegen, The Netherlands, 1991. 
